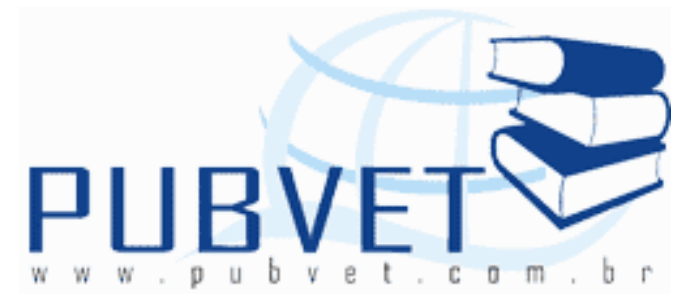

PUBVET, Publicações em Medicina Veterinária e Zootecnia.

\title{
Vascularização arterial do timo em galinha caipira (Gallus gallus)
}

Rozana Cristina Aranteso*, Angelita das Graças de Oliveira Honoratoº, Renato Souto Severino ${ }^{1}$, Danilo Ferreira ${ }^{2}$, Cheston César Honorato Pereira ${ }^{3}$

- Ms - Médica Veterinária - Faculdade de Medicina Veterinária da Universidade Federal de Uberlândia - UFU

1 Prof. Dr. Programa de Pós-graduação da Faculdade de Medicina Veterinária da Universidade Federal de Uberlândia - UFU.

2 Graduando da Faculdade de Medicina Veterinária da Universidade Federal de Uberlândia - UFU.

3 Prof. Ms - Universidade de Rio Verde - FESURV

* Endereço para correspondência: rozanacristina.arantes@gmail.com

\section{Resumo}

Pesquisou-se a vascularização, o número de lobos tímicos em 30 exemplares de galinhas caipiras (Gallus galllus). Foram preparadas mediante a técnica de injeção arterial, com solução aquosa de neoprene látex a 50\%, corada e posteriormente fixadas em solução aquosa de formol a 10\%. Após as dissecações, observaram-se os lobos tímicos na região cervical, em ambos os antímeros, dispostos dorsolateralmente ao feixe vásculo-nervoso. Sua irrigação foi realizada por ramos das artérias comuns dos nervos vagos direita e esquerda, cutâneas cervicais 
ARANTES, R.C. et al. Vascularização arterial do timo em galinha caipira (Gallus gallus). PUBVET, Londrina, V. 5, N. 14, Ed. 161, Art. 1086, 2011.

ascendentes direita e esquerda, tireóideas craniais direita e esquerda, esofágicas ascendentes direita e esquerda, vertebrais direita e esquerda, vertebral ascendente esquerda, carótidas comuns direita e esquerda e ramos cutâneos direitos e esquerdos. O número de colaterais provenientes destas artérias variaram de 16 a 30 para ambos os antímeros No antímero direito emitiram de 6 a 18 ramos e o contralateral de 5 a 15 ramos. O número de lobos tímicos variaram de 9 a 15, assim distribuídos: o antímero direito mostrou-se com 5 a 8 lobos, e o esquerdo com 4 a 7. Ocorreu diversificação dos pontos de entrada dos ramos arteriais no parênquima tímico, desde a sua extremidade cranial, terço médio e extremidade caudal.

Palavras-chave: galinha caipira, timo, vascularização arterial

\title{
Arterial vascularization of the thymus in the domestic fowl (Gallus gallus)
}

\begin{abstract}
Vascularization, the number of thymic lobes were researched in 30 samples of domestic fowl (Gallus gallus). Were prepared by injection technique of arterial vessels with aqueous solution of latex to $50 \%$, colored, and subsequent fixation in $10 \%$ aqueous solution of formaldehyde. After dissection, were observed cervical thymic lobes, in both sides, willing dorsolateral to the vasculo-nervous beam. It's irrigation was performed by common artery branches of the vagus nerve left and right, ascending cervical cutaneous right and left, right and left cranial thyroid, ascending esophageal right and left, right and left vertebral, left ascending vertebral, right and left common carotid and cutaneous branches right and left. The co-lateral from these arteries varied from 16 to 30 for both antimeres. In the right side, issued from 6 to 18 branches and in opposite side, 5 to 15 branches. The number of thymic lobes ranged from 9 to 15, distributed as follows: the right
\end{abstract}


ARANTES, R.C. et al. Vascularização arterial do timo em galinha caipira (Gallus gallus). PUBVET, Londrina, V. 5, N. 14, Ed. 161, Art. 1086, 2011.

side had 5 to 8 lobes, whereas the left had 4 to 7 . There was diversification of the entry points of the branches in the thymic parenchyma, from the cranial extremity, middle third and caudal extremity.

Keyword: Arterial vascularization, domestic fowl, thymus

\section{INTRODUÇÃO}

O atual interesse por um mercado consumidor voltado a produtos orgânicos, coloca a criação de galinhas caipiras num patamar elevado. Sendo considerada uma atividade especifica de avicultura, especialmente pela maneira extensiva de sua exploração e deficiências, no que diz respeito a manejos (sanitário, nutricional e reprodutivo) das aves. Atualmente este segmento tem-se mostrado rentável já que é detentor de um mercado consumidor promissor (BARRETO et al., 2007).

O timo, órgão epitelial derivado da terceira bolsa faríngea, encontrado no mediastino anterior, apresenta-se formado por lobos contendo grupos frouxos de células epiteliais cobertos por cápsula de tecido conjuntivo. É de suma importância nas aves e mamíferos, por ser colonizado pelos linfócitos $T$, tornando o principal local de produção e diferenciação destas células. As aves são os únicos animais, em que o timo permanece funcional por um período mais prolongado, se comparado às outras espécies, em que a regressão ocorre até o final do primeiro ano de vida, o que por certo facilita a sua utilização como protótipo de diferentes pesquisas (WOODARD, 2006).

Objetivam-se com a presente investigação, aprimorar o conhecimento morfológico do timo em galinhas caipiras, no que diz respeito ao número, a origem e posição dos ramos arteriais, a topografia e morfologia dos seus lobos tímicos. 
ARANTES, R.C. et al. Vascularização arterial do timo em galinha caipira (Gallus gallus). PUBVET, Londrina, V. 5, N. 14, Ed. 161, Art. 1086, 2011.

\section{REVISÃO DE LITERATURA}

Numidea meleagris galeata, a popular galinha de Angola, teve seus lobos tímicos investigados por Santana et al. (2007). Após a dissecação, tiveram descritos sua topografia, morfologia e biometria. Cadeias tímicas foram identificadas, na região cervical, com morfologias irregulares e diferentes, nos antímeros contralaterais. Observaram íntima relação com a artéria comum do nervo vago. Contudo consideraram os arranjos tímicos semelhantes a outros galiformes e columbídeos.

Pereira et al. (2008) investigaram timos de galinhas da linhagem Hubbard. Após incisão na linha mediana ventral do pescoço e remoção do osso esterno, os lobos tímicos foram observados quanto a sua vascularização e localização. Encontraram estruturas tímicas desde a primeira vértebra cervical até o limite da cavidade toracoabdominal, com disposição paralela e lateral ao pescoço e proximidade com o nervo vago e veia jugular. Não sendo observado o referido órgão na cavidade toracoabdominal. Houve variação no número de lobos, da ordem de quatro a 13 e de três a 11 nos antímeros direito e o contralateral, respectivamente. Sua irrigação era realizada por ramos oriundos diretamente das artérias comuns dos nervos vagos direita e esquerda, esofágicas ascendentes direita e esquerda, tireóideas craniais direita e esquerda, caudais direita e esquerda e cervicais cutâneas ascendentes direita e esquerda.

Exemplares galináceos da linhagem Paraíso Pedrês, tiveram suas cadeias tímicas descritas por Santana et al. (2008). Observaram o referido órgão desde a fúrcula até próximo a mandíbula, na região cervical, dorsolateral ao plexo neuro vascular do pescoço. A irrigação do timo foi descrita como proveniente de ramos das artérias comuns dos nervos vagos direita e esquerda, tireóideas craniais direita e esquerda, tireóideas caudais direita e esquerda, ingluviais craniais direita e esquerda, ingluviais caudais direita e esquerda, ingluvial esquerda, esofágica ascendente esquerda e ingluvial média direita, e carótida comum direita. 
ARANTES, R.C. et al. Vascularização arterial do timo em galinha caipira (Gallus gallus). PUBVET, Londrina, V. 5, N. 14, Ed. 161, Art. 1086, 2011.

Observaram ainda através de modelos vasculares por corrosão, o comportamento dos ramos arteriais no parênquima dos lobos tímicos, e concluíram que a cortical é bastante vascularizada em contraste com a medular com poucos capilares. As lobulações tímicas apresentaram uma variação de dois a 10 e de três a oito lobos respectivamente antímeros esquerdo e direito.

Lima et al. (2009) caracterizaram o timo na linhagem de aves Máster Gris Cou Plumé. Topograficamente, o mesmo alojava-se nas regiões cervical e torácica, com prevalência de cinco a 11 e quatro a nove lobos, respectivamente nos antímeros esquerdo e contralateral. Cujo suprimento de sangue oxigenado originou-se de ramos diretos e indiretos das artérias ingluviais direita e esquerda, tireóideas craniais direita e esquerda, comuns dos nervos vagos direita e esquerda, artérias carótida comum esquerda, ingluvial, tireóidea caudal esquerda, esofágicas ascendentes direita e esquerda, occipitais direita e esquerda e ramos cutâneos direito e esquerdo. O referido órgão apresentava morfologias variadas.

\section{MATERIAL E MÉTODOS}

Utilizou-se 30 galinhas caipiras (Gallus gallus) com idade entre oito e 20 semanas, provenientes de propriedades rurais dos municípios de Santa Vitória e Ituiutaba, Estado de Minas Gerais - Brasil.

Os animais foram obtidos após morte natural. Injetaram nas aves, através de seu sistema arterial, uma solução de neoprene látex ${ }^{1} \operatorname{corada}^{2}$ a $50 \%$, por meio da canulação da artéria isquiática direita, com imediata fixação das peças após aplicações intramusculares profundas, subcutâneas e intracavitária de solução

\footnotetext{
${ }^{1}$ Du Pont do Brasil S. A. - Indústrias Químicas.

${ }^{2}$ Globo S. A. - Tintas e Pigmentos.
} 
ARANTES, R.C. et al. Vascularização arterial do timo em galinha caipira (Gallus gallus). PUBVET, Londrina, V. 5, N. 14, Ed. 161, Art. 1086, 2011.

aquosa a $10 \%$ de formol $^{3}$. As peças permaneceram submersas nesta mesma solução por um período mínimo de 72 horas para fixação completa, em recipiente adequado.

Após este período, as aves foram dissecadas por meio de instrumentos cirúrgicos adequados, utilizando quanto necessário campo visual de lupa monocular ${ }^{4}$. A dissecação deu-se através de uma incisão mediana longitudinal da pele ventral do pescoço, rebatendo-a dorsolateralmente, e retirando os tecidos adiposo e conjuntivo, para a devida abordagem do timo, com ênfase as artérias que irrigam seus lobos, evidenciando ainda a sua topografia, morfologia e posição dos ramos penetrantes.

Totalizada a dissecação, algumas peças foram fotografadas com finalidade ilustrativa. Os resultados foram submetidos ao Teste Kruskal - Wallis (SAMPAIO, 1998).

Esta pesquisa foi submetida e aprovada pelo Comitê de Ética do Uso de Animais em Pesquisa - Análise final no 074/10 do Comitê de Ética na Utilização de Animais para o Protocolo Registro CEUA/UFU 028/10.

\section{RESULTADOS}

Os lobos tímicos foram observados na região cervical, nas 30 galinhas caipiras $(100 \%)$, desde a terceira vértebra cervical até a entrada da cavidade celomática, em posição dorsolateral ao pescoço. Apresentavam-se como lobos individualizados, com morfologias diversas, variando de alongados, fusiformes e ovalados, e sendo os lobos craniais menores que os caudais. Encontravam-se envolvidos, juntamente com a artéria comum do nervo vago, nervo vago, veia jugular, por tecido adiposo e conjuntivo, nos dois antímeros Já os lobos tímicos

\footnotetext{
3 Química Anastásio Ltda.

${ }^{4}$ Lupa monocular tipo Wild (10x)
} 
ARANTES, R.C. et al. Vascularização arterial do timo em galinha caipira (Gallus gallus). PUBVET, Londrina, V. 5, N. 14, Ed. 161, Art. 1086, 2011.

situados caudalmente apresentaram envolvidos pelos sacos. Os mesmos relacionam-se, no antímero direito, ventralmente com esôfago e inglúvio, e na posição medial-esquerda com a traquéia, nas 30 aves (100\%). As glândulas tireóideas de 5 espécimes (16,66\%) encontravam-se aderidas aos lobos tímicos no antímero esquerdo. Os resultados seguem nas tabelas abaixo (Tabelas 1, 2, 3, $4,5$ e 6$)$.

O teste de Kruskal - Wallis em relação ao número de lobos tímicos nos dois antímeros foi igual a 0,0349, para um nível de significância de $p<0,05$, demonstrando que não houve diferenças estatísticas entre o número de lobos tímicos.

TABELA 1: Número de lobos tímicos independentes do antímero. Uberlândia, MG - 2010.

\begin{tabular}{c|c|c}
\hline Lobos tímicos & $\mathrm{n}^{0}$ de aves & $\%$ \\
\hline 9 & 5 & 16,66 \\
\hline 10 & 1 & 3,33 \\
11 & 6 & 20,00 \\
12 & 3 & 10,00 \\
13 & 3 & 10,00 \\
14 & 5 & 16,66 \\
15 & 7 & 23,33 \\
& 30 & 99,98 \\
\hline
\end{tabular}

TABELA 2: Número de lobos tímicos de acordo com os antímeros direito e esquerdo. Uberlândia, MG - 2010.

\begin{tabular}{c|cc|c|c|c}
\hline \multirow{2}{*}{$\begin{array}{c}\text { Lobos } \\
\text { tímicos }\end{array}$} & \multicolumn{2}{|c|}{ Ant. direito } & Lobos & \multicolumn{2}{c}{ Ant. esquerdo } \\
& $\mathrm{n}^{\circ}$ de aves & $\%$ & tímicos & $\mathrm{n}^{\circ}$ de aves & $\%$ \\
\hline 5 & 6 & 20,00 & 4 & 5 & 16,66 \\
6 & 9 & 30,00 & 5 & 7 & 23,33 \\
7 & 8 & 26,66 & 6 & 6 & 20,00 \\
8 & 7 & 23,33 & 7 & 12 & 40,00 \\
& 30 & 99,99 & & 30 & 99,99 \\
\hline
\end{tabular}


ARANTES, R.C. et al. Vascularização arterial do timo em galinha caipira (Gallus gallus). PUBVET, Londrina, V. 5, N. 14, Ed. 161, Art. 1086, 2011.

TABELA 3: Número de artérias para os antímeros direito e esquerdo. Uberlândia, MG - 2010.

\begin{tabular}{c|c|c|c|c}
\hline \multirow{2}{*}{ Artérias } & \multicolumn{2}{|c|}{ Ant. direito } & \multicolumn{2}{c}{ Ant. esquerdo } \\
\cline { 2 - 5 } & $\mathrm{n}^{\circ}$ de aves & $\%$ & $\mathrm{n}^{0}$ de aves & $\%$ \\
\hline Carótida comum & 4 & 13,33 & 2 & 6,66 \\
Esofágica asc. & 7 & 23,33 & 7 & 23,33 \\
Tireóidea cranial & 8 & 26,66 & 7 & 23,33 \\
Vertebral & 11 & 36,66 & 8 & 26,66 \\
Vertebral asc. & & & 6 & 20,00 \\
Comum n.vago & 30 & 100,00 & 30 & 100,00 \\
Cervical cut. & 30 & 100,00 & 30 & 100,00 \\
asc. & & & & 100,00 \\
Ramos cut. & 30 & 100,00 & 30 & \\
pesc. & & & & \\
\hline
\end{tabular}

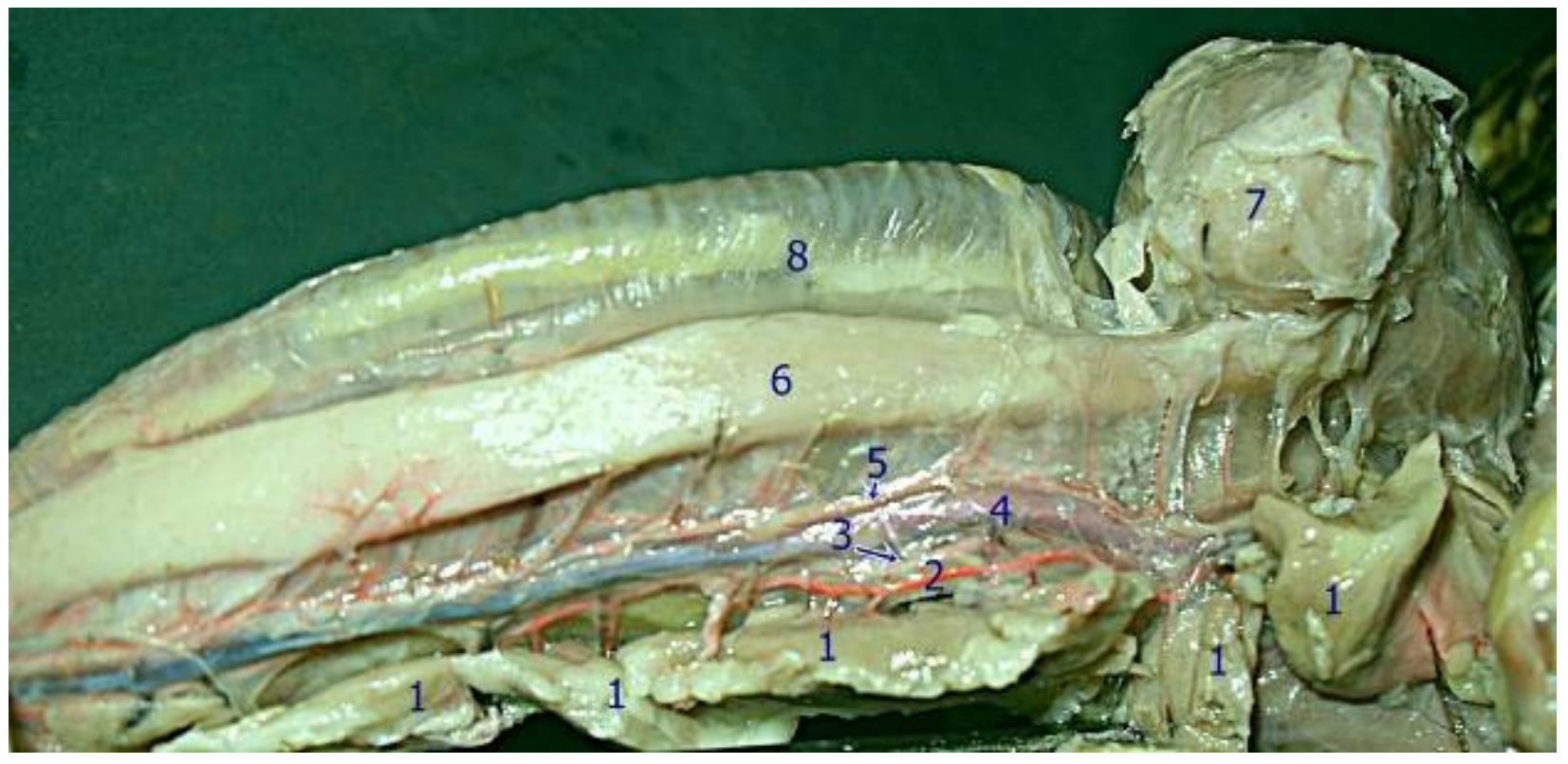

Figura 1. Região lateral direita do pescoço de galinha caipira, (Gallus gallus), com visualização dos lobos tímicos (1), da artéria comum do nervo vago (2), do nervo vago (3), da veia jugular (4) e artéria cutânea cervical ascendente (5). Mostrando ainda, o esôfago (6), o inglúvio (7) e a traquéia (8) 
ARANTES, R.C. et al. Vascularização arterial do timo em galinha caipira (Gallus gallus). PUBVET, Londrina, V. 5, N. 14, Ed. 161, Art. 1086, 2011.

TABELA 4: Distribuição do número de ramos para os antímeros direito e esquerdo. Uberlândia, MG $-2010$.

\begin{tabular}{|c|c|c|c|c|c|c|}
\hline \multirow[b]{2}{*}{ Artérias } & \multicolumn{3}{|c|}{ Ant. direito } & \multicolumn{3}{|c|}{ Ant. esquerdo } \\
\hline & $\begin{array}{c}\mathrm{n}^{\mathrm{O}} \\
\text { ramos }\end{array}$ & $\begin{array}{l}\text { no de } \\
\text { aves }\end{array}$ & $\%$ & no ramos & $\begin{array}{l}\text { no de } \\
\text { aves }\end{array}$ & $\%$ \\
\hline \multirow[t]{2}{*}{ Carótida comum } & 1 & 2 & 6,66 & 1 & 2 & 6,66 \\
\hline & 2 & 2 & 6,66 & & & \\
\hline \multirow[t]{4}{*}{ Esofágica asc. } & 1 & 2 & 6,66 & 1 & 3 & 10,00 \\
\hline & 2 & 1 & 3,33 & 2 & 2 & 6,66 \\
\hline & 3 & 2 & 6,66 & 3 & 2 & 6,66 \\
\hline & 4 & 2 & 6,66 & & & \\
\hline \multirow[t]{2}{*}{ Tireóidea cranial } & 1 & 6 & 20,00 & 1 & 4 & 13,33 \\
\hline & 2 & 2 & 6,66 & 2 & 3 & 10,00 \\
\hline \multirow[t]{2}{*}{ Vertebral } & 1 & 7 & 23,33 & 1 & 5 & 16,66 \\
\hline & 2 & 4 & 13,33 & 2 & 3 & 10,00 \\
\hline \multirow[t]{2}{*}{ Vertebral asc. } & & & & 1 & 4 & 13,33 \\
\hline & & & & 2 & 2 & 6,66 \\
\hline \multirow[t]{6}{*}{ Comum n.vago } & 7 & 9 & 30,00 & 7 & 8 & 26,66 \\
\hline & 8 & 10 & 33,33 & 9 & 10 & 33,33 \\
\hline & 9 & 5 & 16,66 & 11 & 6 & 20,00 \\
\hline & 11 & 3 & 10,00 & 12 & 6 & 20,00 \\
\hline & 12 & 2 & 6,66 & & & \\
\hline & 14 & 1 & 3,33 & & & \\
\hline \multirow{3}{*}{$\begin{array}{c}\text { Cervical cut. } \\
\text { asc. }\end{array}$} & 1 & 12 & 40,00 & 1 & 10 & 33,33 \\
\hline & 2 & 10 & 33,33 & 2 & 13 & 43,33 \\
\hline & 3 & 8 & 26,66 & 3 & 7 & 23,33 \\
\hline \multirow{5}{*}{$\begin{array}{c}\text { Ramos cut. } \\
\text { pesc. }\end{array}$} & 2 & 4 & 13,33 & 1 & 6 & 20,00 \\
\hline & 3 & 4 & 13,33 & 2 & 10 & 33,33 \\
\hline & 4 & 6 & 20,00 & 4 & 5 & 16,66 \\
\hline & 5 & 10 & 33,33 & 6 & 4 & 13,33 \\
\hline & 6 & 6 & 20,00 & 7 & 5 & 16,66 \\
\hline
\end{tabular}


ARANTES, R.C. et al. Vascularização arterial do timo em galinha caipira (Gallus gallus). PUBVET, Londrina, V. 5, N. 14, Ed. 161, Art. 1086, 2011.

TABELA 5: Número de ramos penetrantes por lobo tímico. Uberlândia, MG - 2010.

\begin{tabular}{c|c|c}
\hline $\begin{array}{c}\text { Ramos/ } \\
\text { lobo }\end{array}$ & $\begin{array}{c}\mathrm{n}^{\circ} \text { de } \\
\text { aves }\end{array}$ & $\%$ \\
\hline 16 & 1 & 3,33 \\
17 & 2 & 6,66 \\
18 & 3 & 10,00 \\
19 & 1 & 3,33 \\
20 & 3 & 10,00 \\
21 & 6 & 20,00 \\
22 & 2 & 6,66 \\
23 & 1 & 3,33 \\
24 & 1 & 3,33 \\
26 & 3 & 10,00 \\
27 & 3 & 10,00 \\
30 & 4 & 13,33 \\
\hline
\end{tabular}

TABELA 6: Distribuição dos ramos penetrantes nos antímeros direito e esquerdo. Uberlândia, MG $-2010$.

\begin{tabular}{c|c|c|c|c}
\hline \multirow{2}{*}{ Ramos/lobo } & \multicolumn{2}{|c|}{ Ant. direito } & \multicolumn{2}{c}{ Ant. esquerdo } \\
\cline { 2 - 5 } & $\mathrm{n}^{0}$ de aves & $\%$ & $\mathrm{n}^{0}$ de aves & $\%$ \\
\hline 5 & & & 2 & 6,66 \\
6 & 1 & 3,33 & 2 & 6,66 \\
7 & 1 & 3,33 & 4 & 13,33 \\
8 & & & 4 & 13,33 \\
9 & 3 & 10,00 & 2 & 6,66 \\
10 & 4 & 13,33 & 1 & 3,33 \\
11 & 1 & 3,33 & 3 & 10,00 \\
12 & 2 & 6,66 & 3 & 10,00 \\
13 & 5 & 16,66 & 2 & 6,66 \\
14 & 2 & 6,66 & 6 & 20,00 \\
15 & 3 & 10,00 & 1 & 3,33 \\
16 & 5 & 16,66 & & \\
17 & 2 & 6,66 & & \\
18 & 1 & 3,33 & & \\
\hline
\end{tabular}


ARANTES, R.C. et al. Vascularização arterial do timo em galinha caipira (Gallus gallus). PUBVET, Londrina, V. 5, N. 14, Ed. 161, Art. 1086, 2011.

TABELA 7: Distribuição dos ramos penetrantes para os lobos tímicos nos antímeros direito e esquerdo. Uberlândia, MG - 2010.

\begin{tabular}{c|c|c}
\hline Posição & Ant. direito \% & Ant. esquerdo \% \\
\hline Extremidade cranial & 2,50 & 6,30 \\
Extremidade cranial e caudal & 40,00 & 44,00 \\
Extremidade cranial e caudal & 30,00 & 27,20 \\
e terço médio & & \\
Extremidade caudal e terço & 2,20 & 2,00 \\
$\quad$ médio & & 16,50 \\
Terço médio & 19,00 & 2,90 \\
Extremidade cranial e terço & 4,00 & 1,10 \\
médio & & \\
Extremidade caudal & 2,30 & \\
\hline
\end{tabular}

\section{DISCUSSÃO}

Os lobos tímicos da galinha caipira mostram-se situados lateralmente, nos dois antímeros do pescoço, em íntima relação com as artérias comuns dos nervos vagos, os nervos vagos e as veias jugulares, envolvidos por tecidos adiposo e conjuntivo, achados estes similares com Nickel et al. (1977), King (1986), Santana et al. (2007), Santana et al. (2008) e Lima et al. (2009). Ainda no que diz respeito à topografia tímica o observamos desde a região cervical até a entrada do tórax, estando em concordância com Santana et al. (2007), Pereira et al. (2008) e Santana et al. (2008), os quais caracterizaram o timo desde a primeira vértebra cervical até a entrada da cavidade toracoabdominal. Santana et al. (2008), foram mais específicos destacando o osso fúrcula como o limite caudal da região cervical. Sendo divergente das citações de Lima et al. (2009), que fizeram menção ao timo posicionado além da região cervical, também na região torácica. Presume-se que a topografia tímica nas aves deva-se a diferentes conotações morfofuncionais, como aspecto constitucional, habitat, exploração zootécnica, entre outras. 
ARANTES, R.C. et al. Vascularização arterial do timo em galinha caipira (Gallus gallus). PUBVET, Londrina, V. 5, N. 14, Ed. 161, Art. 1086, 2011.

Sob o aspecto do número de lobos, nas galinhas caipiras, o observamos nos antímeros direito e esquerdo, numa variação numérica de 5 a 8 e 4 a 7 lobos, respectivamente. Nossos resultados mostraram-se diferentes dos achados de Pereira et al. (2008), Santana et al. (2008) e Lima et al. (2009). Ao analisarmos as variações inferiores observamos valores muito próximos. Porém quanto às variações superiores as discrepâncias foram maiores. Estas constatações são próprias, em se tratando de aspectos individuais, em consequência da variabilidade genética especialmente nas diferentes linhagens avícolas.

Quanto às artérias responsáveis pela irrigação dos lobos tímicos na galinha caipira no antímero direito são: carótida comum direita, tireóidea direita, comum do nervo vago direita, ramos cutâneos direitos do pescoço, cervical cutânea ascendente direita, vertebral direita e esofágica ascendente direita. Ao analisarmos os nossos resultados frente ao de outros pesquisadores como Pereira et al. (2008), Santana et al. (2008) e Lima et al. (2009), observamos diferenças interessantes, no atinente à ausência, na galinha caipira, de ramos originados das artérias ingluvial cranial direita e ingluvial média direita (SANTANA et al., 2008), ingluvial caudal direita (SANTANA et al., 2008) e (LIMA et al., 2009), tireóidea cranial direita (PEREIRA et al., 2008), (SANTANA et al., 2008) e (LIMA et al., 2009), tireóidea caudal direita, (PEREIRA et al., 2008) e (SANTANA et al., 2008), occipital direita (LIMA et al., 2009). Ainda, no atinente à irrigação tímica no antímero direito, constatamos que as artérias carótida comum direita, vertebral direita, ramos cutâneos direitos do pescoço, tireóidea direita e esofágica ascendente direita, por nós identificadas nutrindo o timo na galinha caipira não o fazem em outras linhagens avícolas. Assim as ausências das artérias carótida comum direita são retratadas por (PEREIRA et al., 2008) e (LIMA et al., 2009), da vertebral direita por (PEREIRA et al et al., 2008), (SANTANA et al., 2008) e (LIMA et al., 2009), dos ramos cutâneos direito do pescoço e artéria tireóidea direita por 
ARANTES, R.C. et al. Vascularização arterial do timo em galinha caipira (Gallus gallus). PUBVET, Londrina, V. 5, N. 14, Ed. 161, Art. 1086, 2011.

(PEREIRA et al et al., 2008), da esofágica ascendente direita por (SANTANA et al., 2008).

As artérias que fazem o suprimento arterial para os lobos tímicos na galinha caipira, no antímero esquerdo são: carótida comum esquerda, tireóidea esquerda, comum do nervo vago esquerda, ramos cutâneos esquerdos do pescoço, cervical cutânea ascendente esquerda, vertebral esquerda, vertebral ascendente esquerda e esofágica ascendente esquerda. Ao compararmos nossos resultados com as diferentes linhagens ou espécies de aves estudadas por outros pesquisadores, detectamos considerações distintas em relação a não participação na galinha caipira, de ramos originados das artérias: tireóidea cranial esquerda e tireóidea caudal esquerda citadas por (PEREIRA et al., 2008) e (SANTANA et al., 2008). Ao compararmos ainda, outras artérias que se destinam ao timo, no antímero esquerdo da galinha caipira como: artéria tireóidea esquerda, vertebral ascendente esquerda, vertebral esquerda, carótida comum esquerda e cervical cutânea ascendente esquerda, e confrontá-las com os achados de outros pesquisadores estas mostram-se ausentes como a artéria tireóidea esquerda quando retratada por (PEREIRA et al., 2008) e (SANTANA et al., 2008), vertebral ascendente esquerda, vertebral esquerda e carótida comum esquerda por (SANTANA et al., 2008) e (LIMA et al., 2009) e cervical cutânea ascendente por (LIMA et al., 2009). Situações como estas, configuram uma individualidade anatômica própria de diferentes espécies frente aos desafios ambientais.

Em relação ao número de ramos penetrantes no parênquima tímico das galinhas caipiras, independentes de suas origens, notamos variações de 6 a 18, no antímero direito e de 5 a 15 no contralateral. Confrontando nossos resultados com Pereira et al. (2008) verificamos discrepância em relação aos valores mínimos e máximos, visto que contabilizaram de 8 a 25 ramos no direito e 9 a 22 no esquerdo. Tal fato pode estar relacionado com as variabilidades genética, fenotípica e ambiental das diversas linhagens de aves estudadas. 
ARANTES, R.C. et al. Vascularização arterial do timo em galinha caipira (Gallus gallus). PUBVET, Londrina, V. 5, N. 14, Ed. 161, Art. 1086, 2011.

Quanto à entrada das artérias nos lobos tímicos, notamos que, na galinha caipira, existem vários pontos de origem: em sua extremidade cranial; extremidades cranial e caudal; extremidades cranial; terço médio; extremidades cranial, caudal e terço médio; extremidade caudal e terço médio e sua extremidade caudal. As quais estão em consonância com Santana et al. (2008), que discriminaram estes mesmos locais de penetração. Lima et al. (2009), também citaram locais variáveis de penetração dos colaterais, mas sem especificarem as posições. Nossos achados diferem dos de Pereira et al. (2008), que evidenciaram presença de hilo tímico.

O teste de Kruskal - Wallis demonstrou que não houve diferenças estatísticas entre o número de lobos tímicos nos dois antímeros, demonstrando falta de concordância com Pereira et al. (2008), que observaram desigualdade estatística entre os lobos tímicos.

\section{CONCLUSÕES}

Deste estudo pode-se concluir que na galinha caipira, as artérias que irrigam os lobos tímicos apresentam comportamentos bem definidos e com características comuns e diferentes em relação a outras linhagens avícolas, o timo nesta espécie é vascularizado por ramos diretos das artérias comuns dos nervos vagos direita e esquerda, cervicais cutâneas ascendentes direita e esquerda, esofágica esquerda, carótida comum esquerda, vertebral direita, tronco vagovertebral, tireóideas craniais direita e esquerda, com variação numérica de nove a quinze lobos tímicos independentemente do antímero.

\section{REFERÊNCIAS}

BARRETO, C. G.; BEZERRA, M. da C. T.; LORENA, C.; SOUSA, R. B. de; BARROS JÚNIOR, G.; OLIVEIRA, F. G. de. Capacitação de comunidades rurais no Estado da Paraíba em organização do trabalho coletivo e manejo de galinha de capoeira. 2007. Disponível em: http://www.cnpat.embrapa.br/sbsp/anais/Trab_Format_PDF/185.pdf. Acesso em: 02 de out. 2010. 
ARANTES, R.C. et al. Vascularização arterial do timo em galinha caipira (Gallus gallus). PUBVET, Londrina, V. 5, N. 14, Ed. 161, Art. 1086, 2011.

KING, A. S. Sistema Linfático. In: GETTY, R. Sisson/Grossman anatomia dos animais domésticos. 5. ed., Rio de Janeiro: Guanabara, 1986, v. 2, cap. 68, p.1881-1889.

LIMA, E.M. M. de; BRAVO, M. de O.; VIANNA, A. R. da C. B.; CARNEIRO E SILVA, F. O.; SEVERINO, S.R.; DRUMMOND, S.S.; SANTANA, M. I. S. Suprimento vascular arterial do timo em aves (Gallus gallus) da linhagem Máster Gris Cou Plumé. Biotemas, Florianópolis, v. 22, n.3, p. 171-176, set. 2009.

NICKEL, R.; SCHUMMER, A.; SEIFERLE, E.. Anatomy of the Domestic Birds. Berlim: Verlag Paul Parey, 1977, p.85-107.

PEREIRA, C. C. H.; BOMBONATO, P. P.; CARNeiro E SilVA, F. O.; HONORATO, A. das G. De O. Irrigação do timo em aves (Gallus gallus) da linhagem Hubbard. Veterinária Notícias, Uberlândia, v. 14, n. 1, p. 41-47, jan./jun., 2008. Disponível em: <http://www.vetnot.famev.ufu.br/>. Acesso em: 08 mar. 2009.

SANTANA, M. I. S.; BOMBONATO, P. P.; CARNEIRO E SILVA, F. O.; BENEDICTO, H. G. Morfologia e morfometria do timo em galinhas de Angola (Numidea meleagris galeata). Revista Portuguesa de Ciências Veterinárias, Lisboa, Portugal, v. 102, n. 561-562, p. 43-48, 2007. Disponível em: <http://www.fmv.utl.pt/spcv/PDF/pdf6_2007/43-48.pdf>. Acesso em: 08 mar. 2009.

SANTANA, M. I. S.; BOMBONATO, P. P.; ARAúJO, L. V.; CASTELlO, A. P. F.; ESCANHOELA, T. B. Irrigação do timo em aves da linhagem Paraíso Pedrês (Gallus gallus). Arquivos Brasileiros de Medicina Veterinária e Zootecnia, São Paulo, v. 60, n. 2, p. 307-314, 2008. Disponível em: < http://www.scielo.br/pdf/abmvz/v60n2/a05v60n2.pdf>. Acesso em: 07 mar. 2009.

WOODARD, J. C. Sistemas hêmico e linfático. In: JONES, T. C.; HUNT, R. D.; KING, N. W. Patologia Veterinária. 6.ed. São Paulo: Manole, 2006, cap. 22, p. 1027-1061. 